\title{
A Large Filament and Flares in Active Region NOAA 5669 on September 2, 1989
}

\author{
G. A. Porfir'eva and G. V. Yakunina \\ Sternberg Astronomical Institute, 119899 Moscow B-234, Russia \\ and Z. Mouradian \\ Observatoire de Paris-Meudon, URA 326, France
}

\begin{abstract}
The behaviour of extended filaments in the vicinity of a new emerging magnetic flux site has been investigated during the $2 \mathrm{~N} / \mathrm{M} 5.8$ flare on September 1 and several flares on September 2, 1989.
\end{abstract}

\section{Introduction}

The behaviour of flare-associated filaments might be complex and the observational picture becomes complicated for different Doppler velocities along the filament channel. Filtergrams, obtained simultaneously in the center and wings of the $\mathrm{H}_{\alpha}$ line up to $\lambda=H_{\alpha} \pm 3 \AA$, permits us to better understand the filament behaviour and its relation with the flare situation in the active region (AR). The analysis of the evolution of extended filaments helps us to investigate large magnetic arches. In this paper we try to follow the behaviour of the long-lived large filament and filament-like structures in AR NOAA 5669 with a complex magnetic field on September 2, 1989 during the 2N/M5.8 flare and four consecutive subflares, one of which was accompanied by a M1.1-class X-ray burst.

\section{Observations and Description of the AR}

Observations were obtained at the High Altitude Station of Sternberg Astronomical Institute near Alma-Ata, Kazakhztan on a coude-refractor Opton using an $\mathrm{H}_{\alpha}$ filter with a passband of $0.25 \AA$. We used also $\mathrm{H}_{\alpha}$ filtergrams of the Meudon Observatory, photospheric (FeI 5324.19 $)$ and chromocpheric $\left(\mathrm{H}_{\beta} 4861.34 \AA\right)$ magnetograms, $\mathrm{H}_{\beta}$ Dopplergrams and $\mathrm{H}_{\beta}$ images, obtained at Huairou Solar Station (Table 1).

The AR NOAA 5669 was a large complex spot group stretching for about $30^{\circ}$ from east to west. The leading spot, $\mathrm{N} 1$, had negative polarity and the following ones had mainly positive polarity, being surrounded by little spots and pores of both $\mathrm{N}$ and $\mathrm{S}$ polarities. The large-scale magnetic polarities interchanged as N-S-N-S-N-S. A schematic drawing of the AR (Figure 1a) is a result of the superposition of photos, obtained in red and blue wings of the $\mathrm{H}_{\alpha}$ line, and $\mathrm{H}_{\beta}$ chromospheric magnetic charts, recorded at 0155 and $0239 \mathrm{UT}$ (Zhang et al. 1992).

Comparing magnetograms (see Zhang et al. 1992) we notice that a good 


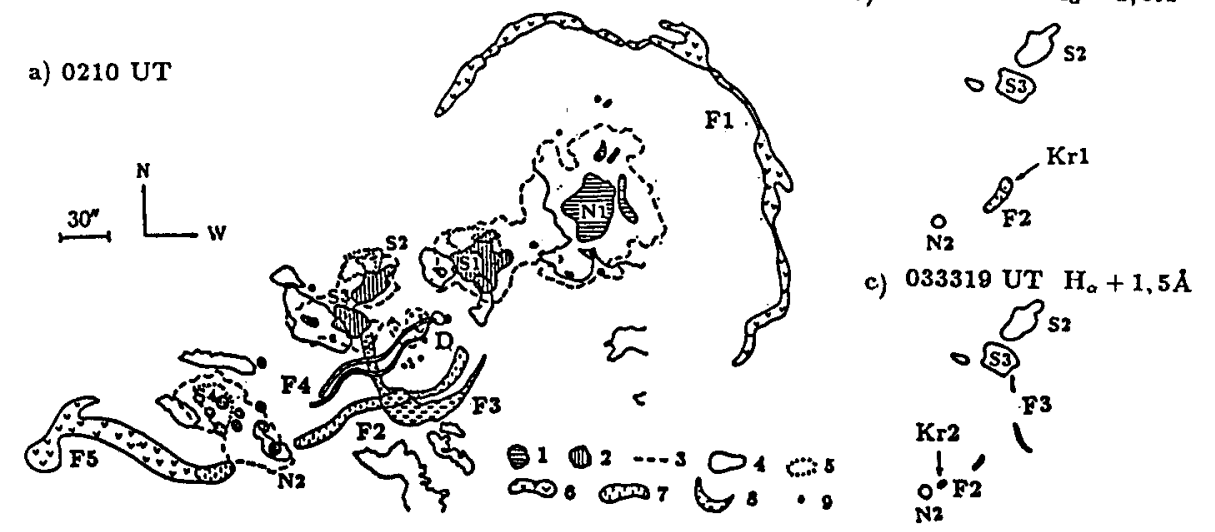

Figure 1. Drawings of events in AR5669: spots of $\mathrm{N}(1)$ and $\mathrm{S}(2)$ polarities, $\left(H_{\alpha} \pm 3 \AA\right.$ ) ; penumbra (3); chromospheric fields of $\mathrm{N}(4)$ and S(5) polarities; filaments in center of $H_{\alpha}(6)$, in $H_{\alpha}-0.75 \AA$ ( (7), in $H_{\alpha}+0.75 \AA$ (8); little spots and pores (9).

similarity existed between the main magnetic features observed at the photospheric and chromospheric levels. Large-scale magnetic structures were relatively stable during several hours. Small-scale elements in the middle of the AR changed dynamically. Here in the vicinity of the $D$ magnetic feature new emerging magnetic flux (EMF) was observed on September 2. Numerous little spots and pores were seen here on the $\mathrm{H}_{\alpha}$ filtergrams, taken at $\lambda=H_{\alpha} \pm 3 \AA$ (Figure 1a). In this place the transverse magnetic field was noticeably sheared. Here at 2350 UT on September 1 the 2N/M5.8 flare occurred and for six hours after it $\mathrm{H}_{\alpha}$ emission kernels of several subflares were located there. In the front the AR was encircled with a large quiet filament, F1, situated on the boundary of the large-scale magnetic field net. The F2 filament, having a length of about $3 \times 10^{5} \mathrm{~km}$, was located beneath the AR along the boundary between the magnetic field regions of $S$ and $N$ polarities. The east end of F2 was near the N2 spot (Figure 1a). The loop-like $\mathrm{H}_{\alpha}$ structure F3 was observed for several hours. It looked most striking at 0209:35 UT in $\lambda=H_{\alpha}+0.75 \AA$. Later its intensity decreased and at $0530 \mathrm{UT}$ it was not seen at all. In the middle of the AR filament-like structures, F4, were visible, located nearly parallel to the F2 filament and ending in the vicinity of the EMF near D. Behind the AR the large filament, F5, existed. One of its ends was anchored in the vicinity of the trailer spot group S4 (Figure 1a). The F2 filament was also observed in the $\mathrm{H}_{\beta}$ line but the $\mathrm{F} 3$ and $\mathrm{F} 4$ structures were not seen in the $\mathrm{H}_{\beta}$ line (Zhang et al. 1992).

\section{Results and Discussion}

The behaviour of the large $\mathrm{H}_{\alpha}$ chromospheric features was investigated during several flares on September 2, 1989 and compared with the magnetic structures and Dopplergrams. The eastern parts of the $\mathrm{H}_{\beta} \mathrm{F} 2$ filament, adjacent to the N2 
Table 1. Observational Data about NOAA 5669

\begin{tabular}{|c|c|c|c|c|c|c|}
\hline \multirow{3}{*}{ Date } & \multicolumn{2}{|c|}{ Magnetic field } & \multirow{2}{*}{$\begin{array}{l}\text { Velocity } \\
\text { field }\end{array}$} & \multirow{2}{*}{$\begin{array}{c}\bar{H}_{\beta} \\
\text { images }\end{array}$} & \multirow{3}{*}{$\begin{array}{c}H_{\alpha} \\
\text { filtergram } \\
\text { (Alma-Ata) }\end{array}$} & \multirow{3}{*}{$\begin{array}{l}\text { Events } \\
\text { in AR } \\
\text { (SGD) }\end{array}$} \\
\hline & photosph. & chromosph. & & & & \\
\hline & \multicolumn{4}{|c|}{ Zhang et al. 1992} & & \\
\hline $1 . \mathrm{IX}$ & & & & 2250 & & \\
\hline 1.IX & & & 2351 & $\overline{2354}$ & & $2350-2502$ \\
\hline $2 . I X$ & 0002 & 0042 & 0032 & 0025 & & $2 \mathrm{~N} / \mathrm{M} 5.8$ \\
\hline $2 . \mathrm{IX}$ & 0107 & 0154 & & 0154 & $\begin{array}{c}0150 \\
0208-0210\end{array}$ & $\begin{array}{c}0141-0156 \text { SF/M1.1 } \\
0153-0227 \text { SID }\end{array}$ \\
\hline $2.1 \mathrm{X}$ & & 0238 & 0235 & 0238 & & $0231-0242 \mathrm{SF}$ \\
\hline $2 . \mathrm{IX}$ & & 0300 & & & $0309-0315$ & $H_{\alpha}$-brightening \\
\hline 2.IX & 0335 & 0338 & 0325 & 0333 & $0330-0336$ & $0330-0345 \mathrm{SN}$ \\
\hline $2 . \mathrm{IX}$ & 0544 & 0534 & 0527 & 0530 & $0524-0536$ & $0522-0546 \mathrm{SF}$ \\
\hline
\end{tabular}

spot, were seen during the preflare phase, maximum and decay of the 2N/M5.8 flare on September 1, 1989 and were not observed in the vicinity of the flare site (Zhang et al. 1992). The large S-shaped F5 filament, located just behind the AR, had a similar behaviour (Porfir'eva and Yakunina 1995). It became active when flares occurred near the filament end in the vicinity of the singularity pivot-point, situated near the S4 spot group (Figure 1a). This pivot point in the AR was found by Mouradian and Soru-Escout (1991).

During the five hours after $0100 \mathrm{UT}$, the F2 filament was observed along its whole channel length both in the $\mathrm{H}_{\beta}$ line and the $\mathrm{H}_{\alpha}$ line. Comparison of the $\mathrm{H}_{\alpha}$ filtergrams with the $\mathrm{H}_{\beta}$ images has shown that there was a good match between the contours of the $\mathrm{H}_{\alpha}$ and $\mathrm{H}_{\beta} \mathrm{F} 2$ filament. The sites with the maximum $\mathrm{H}_{\alpha}$ intensities corresponded to the places with the maximum $\mathrm{H}_{\beta}$ intensities. The $\mathrm{H}_{\alpha}$ F2 filament was active during the observations (see Table 2, for the wavelengths of maximum intensities of the filaments). The topology of Doppler velocities

Table 2. Events in the AR NOAA 5669 on September 2, 1989.

\begin{tabular}{|c|c|c|c|c|c|}
\hline $\begin{array}{c}\text { Universal } \\
\text { time }\end{array}$ & $\begin{array}{l}\text { Data about } \\
\text { flares (SGD) }\end{array}$ & $\begin{array}{c}\text { Filament } \\
\text { F2 }\end{array}$ & $\begin{array}{c}\text { Loop } \\
\text { structure F3 }\end{array}$ & $\begin{array}{c}\text { Filament } \\
\text { F4 }\end{array}$ & $\begin{array}{c}\text { Flare } \\
\text { locations }\end{array}$ \\
\hline $0208-0210$ & $\begin{array}{c}0154 \mathrm{M} 1.1 \\
0153-0227 \text { SID }\end{array}$ & $H_{\alpha}-0.5 \AA$ & $\begin{array}{c}H_{\alpha}+0.75 \AA \\
H_{\alpha}+1 \AA\end{array}$ & $H_{\alpha}-0.5 \AA$ & \multirow{4}{*}{$\begin{array}{c}\text { Emission } \\
\text { kernels in } \\
\text { back part } \\
\text { of the AR } \\
\text { and in } \\
\text { region of } \\
\text { emergent } \\
\text { magnetic } \\
\text { flux } \\
\text { near D }\end{array}$} \\
\hline $0309-0315$ & $H_{\alpha}$-brightening & $\begin{array}{c}H_{\alpha}-0.75 \AA \\
\text { Kr1 in } H_{\alpha}-1.5 \AA \\
\text { in east part of } \mathrm{F} 2 \\
\mathrm{Kr} 2 \text { in } H_{\alpha}+0.75 \AA \\
\text { near the spot } \mathrm{S} 5\end{array}$ & $\begin{array}{l}H_{\alpha}+0.75 \AA \\
\text { vestiges } \\
\text { in } H_{\alpha}+1 \AA\end{array}$ & $\begin{array}{c}\tilde{\mathrm{H} \alpha} \\
H_{\alpha}-0.75 \AA\end{array}$ & \\
\hline $0330-0336$ & $\begin{array}{c}0330-0334-0345 \\
\text { SN 21S, } 54 \mathrm{E}\end{array}$ & $\begin{array}{c}H_{\alpha}-0.75 \bar{A} \\
\mathrm{Kr} 1 \text { in } H_{\alpha}-1.5 \AA \\
\text { in east part of } \mathrm{F} 2 \\
\mathrm{Kr} 2 \text { in } H_{\alpha}+0.75 \AA \\
\text { near the spot } \mathrm{S} 5\end{array}$ & $\begin{array}{r}H_{\alpha}+0.75 \AA \\
\text { vestiges } \\
\text { in } H_{\alpha}+1.5 \AA\end{array}$ & $\begin{array}{c}\bar{H}_{\alpha} \\
H_{\alpha}-0.75 \AA\end{array}$ & \\
\hline $0534-0536$ & $\begin{array}{c}0522-0524-0546 \\
\text { SN 18S, 48E }\end{array}$ & $\begin{array}{c}H_{\alpha}-0.5 A_{;} \\
\mathrm{Kr} 1 \text { in } H \alpha-1 \AA \\
\text { in east part of } F 2\end{array}$ & & $\mathrm{H} \alpha-0.5 \AA$ & \\
\hline
\end{tabular}


along F2 was complex and changeable. A correlation between $\mathrm{H}_{\alpha}$ filtergrams and $\mathrm{H}_{\beta}$ Dopplergrams existed. Preferential blue Doppler shifts were registered along the $\mathrm{H}_{\beta}$ filament at 0235,0325 and 0527 UT. Before a subflare at 0330 UT red Doppler shifts were observed in F2 near the N2 spot (Zhang et al, 1992). The filament looked more intensive in the blue $H_{\alpha}$ wings, and it was seen along its whole length in $\lambda=H_{\alpha}-1 \AA$, which agrees in general with the $\mathrm{H}_{\beta}$ velocity. The maximum Doppler velocity was in kernel $\mathrm{Kr} 1$ (Figure $1 \mathrm{~b}$, Table 2). At 0309-0336 UT a little site $\mathrm{Kr} 2$, seen in the red wing of the $\mathrm{H}_{\alpha}$ line from $\lambda=H_{\alpha}+0.75 \AA$ to $\lambda=H_{\alpha}+2 \AA$, existed near the N2 spot. Hence, we conclude that both in the $\mathrm{H}_{\alpha}$ filtergrams and $\mathrm{H}_{\beta}$ Dopplergrams, red Doppler shifts existed in the eastern part of F2. At the same time blue shifts dominated the western parts of F2 (Figure 1b, c, Table 2).

Distinct filament-like structures $\mathrm{P} 1, \mathrm{P} 2$ and $\mathrm{T}$ are noticeable on the chromospheric magnetic charts at 0154 and 0534 UT (Zhang et al. 1992). The magnetic structures $P 1$ and $T$ coincided by their location with the eastern and western parts of the F2 filament. The third structure P2 is possible to compare with the $\mathrm{H}_{\alpha}$ features F4 (Figure 1a). As was indicated by Zhang et al. (1992), the appearance of the magnetic structures $\mathrm{P} 1$, P2 and $\mathrm{T}$ likely reflected the magnetic field configuration inside the filaments F2 and F4.

The AR looked active when sudden ionospheric disturbances (Table 1) were observed. A distinctive feature in the AR was the large loop-like structure F3, having a shape of a flag in $\lambda=H_{\alpha}+0.75 \AA$ (Figure 1a). A leg was anchored in the S3 spot. The F3 feature remained visible until $0335 \mathrm{UT}$. This magnetic feature possibly existed before $0200 \mathrm{UT}$ and was visible in the $\mathrm{H}_{\beta}$ flare.

\section{Summary}

The behaviour of the large filaments in the vicinity of the EMF in the middle of AR 5669 was analysed during several hours after the $2 \mathrm{~N} / \mathrm{M} 5.8$ flare on September 1, 1989 (2350 UT). The filaments were active during the entire time of the observations and had Doppler velocities equal to dozens of $\mathrm{km} \mathrm{s}^{-1}$. The contours of the $\mathrm{H}_{\alpha}$ and $\mathrm{H}_{\beta} \mathrm{F} 2$ filament and their locations were similar. A definite correlation between $\mathrm{H}_{\alpha}$ Doppler shifts in the F2 filament and $\mathrm{H}_{\beta}$ Dopplergrams existed. The observed activation could be explained by a gradual decay of the $2 \mathrm{~N} / \mathrm{M} 5.8$ flare, accompanied by several subflares. The investigated filaments were long-lived features. The $\mathrm{H}_{\alpha}$ Meudon filtergrams show that the filaments F2 and F3 stayed visible all during the AR's passage across the solar disk.

\section{References}

Mouradian Z. and Soru-Escout I. 1991, in Dynamics of Solar Flares, B. Schmieder and E. Priest (eds.), Observ. de Paris, DASOP, p. 139

Porfir'eva G.A. and Yakunina G.V. 1995, Izvestia Academia Nauk., 59, 181

Zhang H., Ai G., Li W. and Chen J. 1992, Sol. Phys., 146, 61 